\title{
Cardiovascular monitoring with acetylcholinesterase inhibitors: a clinical protocol ${ }^{\dagger}$
}

\author{
Jeremy P. Rowland, John Rigby, Adam C. Harper \\ \& Rosalind Rowland
}

Abstract There has been significant anxiety among prescribers regarding the potential for cardiac adverse effects associated with acetylcholinesterase (AChE) inhibitors in Alzheimer's disease. There is no consensus on how to manage this cardiovascular risk, and memory clinics vary widely in their practice. Review of published evidence reveals that the incidence of cardiovascular side-effects is low, and that serious adverse events are rare. Intensive cardiovascular screening such as pre-treatment electrocardiograms or $24 \mathrm{~h}$ cardiac monitoring is not justified. Furthermore, there are no high-risk groups to target. This article suggests pragmatic guidelines for managing cardiovascular risk in patients receiving AChE inhibitors. The guidelines are intended to be easy to incorporate into routine clinical practice in a memory clinic.

A few years ago it was estimated that almost 18 million people worldwidehad dementia (Alzheimer's Society, 2004), with Alzheimer's disease accounting for over half of cases (Fratiglioni, 2000). The secondgeneration acetylcholinesterase (AChE) inhibitors donepezil, rivastigmine and galantamine were introduced into clinical practice from 1997 for the symptomatic treatment of Alzheimer's disease, and are in wide use. Donepezil alone has had over 770 million days of patient use (Jackson et al, 2004).

The risk of significant and potentially serious cardiovascular side-effects of AChE inhibitors was highlighted from the outset, and product information stated that they have a vagotonic effect on the heart. Although product information for all three drugs gave low rates of cardiovascular adverse effects (Table 1 ), sick sinus syndrome and cardiac conduction defects were quoted as relative contraindications to their use. The manufacturers of donepezil, rivastigmine and galantamine (under the trade names Aricept, Exelon and Reminyl respectively) also advised that the drugs should be used with caution in patients taking concurrent medications that reduce heart rate such as digoxin and beta-blockers. There

${ }^{+}$For a commentary on this article see pp. 185-186, this issue. thus followed some uncertainty as to how treatment should be properly managed.

Since the manufacturers' cautions remain (see the relevant entries in http://emc.medicines.org.uk) and guidance has been unforthcoming, services now vary widely in their practice: some, for example, require electrocardiograms (ECGs) before use and during treatment, whereas others do not. A middle position, with monitoring only of what are considered at-risk groups, is probably the most common approach. It is clear, however, that there is no consensus.

This article arose from the need to develop a simple approach to managing cardiovascular risk in patients with Alzheimer's disease attending a memory clinic for the prescription of $\mathrm{AChE}$ inhibitors. In preparing

Table 1 Frequency of cardiovascular adverse effects for donepezil, galantamine and rivastigmine ${ }^{1}$

Adverse effect Frequency, \%

Dizziness and syncope

Bradycardia, atrial arrhythmias, $0.01-1$ myocardial infarction, angina, seizures

Sino-atrial and atrioventricular block $\quad$ 0.001-0.1

1. Sources: Shire Pharmaceuticals Ltd, 2005; Eisai Ltd, 2006; Novartis Pharmaceuticals UK Ltd, 2007.

Jeremy Rowland is a senior house officer in old age psychiatry based at the Hesketh Centre (Merseycare NHS Trust, 51-55 Albert Road, Southport, Merseyside PR9 0LT, UK. Email: jezrowland@yahoo.com). He moved into psychiatry after initially training in general medicine and gaining membership of the Royal College of Physicians. John Rigby is a consultant psychiatrist and clinical director of the Older People's Mental Health Services in Mersey Care NHS Trust. Adam Harper is a consultant geriatrician for Brighton and Sussex University Hospitals NHS Trust. Rosalind Rowland is a general physician currently studying at the Liverpool School of Tropical Medicine. 
appropriate guidelines we reviewed the incidence and severity of cardiovascular adverse events described in the original randomised controlled trials with donepezil, galantamine and rivastigmine, as well as subsequent open-label studies, and experience from case reports. We asked ourselves the following questions: does currently available evidence support the need for cardiovascular screening and monitoring when using AChE inhibitors? What screening tool should be used? Should screening apply to all patients receiving treatment, or just be targeted at high-risk groups?

\section{Therapeutic use of $\mathrm{AChE}$ inhibitors in Alzheimer's disease}

The core neurochemical abnormality seen in Alzheimer's disease is reduced central cholinergic activity (Giacobini, 1990). Degeneration of nuclei in the basal forebrain results in a loss of excitatory cholinergic transmission to the cerebral cortex, and this is thought to contribute significantly to the cognitive deficits seen (Francis et al, 1999). This has formed the rationale for the use of AChE inhibitors as a therapeutic intervention since they serve to reduce the breakdown of endogenously produced acetylcholine in synaptic clefts, thus improving cholinergic function (Evans et al, 2004).

\section{Potential for cardiovascular adverse events}

First-generation AChE inhibitors were limited in their use owing to severe peripheral cholinergic effects and potentially serious cardiovascular and hepatotoxic adverse effects (Davis et al, 1992; Farlow et al, 1992; Knapp et al, 1994). Second-generation AChE inhibitors, including donepezil, galantamine and rivastigmine, demonstrate a similar benefit but grant a more favourable clinical profile. This is thought to be due to their relative selectivity towards central rather than peripheral cholinesterase inhibition (Jackson et al, 2004).

Parasympathetic activity dominates neural control of heart rate and cardiac function. ${ }^{1}$ Increased levels of acetylcholine stimulate GABAergic and glycinergic

\footnotetext{
1. Interpretation of ECGs is discussed in Abdelmawla, N. \& Mitchell, A. (2006) Sudden cardiac death and antipsychotics. Part 2: Monitoring and prevention. Advances in Psychiatric Treatment, 12, 100-109. Heart block and general heart function are discussed in O'Brien, P. \& Oyebode, F. (2003) Psychotropic medication and the heart. Advances in Psychiatric Treatment, 9, 414-423 (both articles may be accessed free from http:/ / apt. rcpsych.org). Ed.
}

inhibitory receptors by triggering vagal neurotransmission (Wang et al, 2003), and this vagal activity, via muscarinic receptors, acts to slow heart rate. Therefore, theoretically, AChE inhibitors can induce sinus bradycardia, sino-atrial block, and aggravate pre-existing sinus node disease and atrioventricular block (Savie et al, 1998) (Box 1). In support of this theory, donepezil overdose has been reported to result in profound sinus bradycardia that can be reversed with atropine (Shepherd et al, 1999; Hidetaka et al, 2003). Furthermore, edrophonium, an anticholinesterase, has been shown to have a strong ability to induce syncope during head-up tilt-testing (Fitzpatrick et al, 1996). Finally, heart rate variability as a measure of autonomic control has been shown to be significantly decreased in patients who are taking donepezil, although this result was not compared with a placebo (McLaren et al, 2003).

The critical question is the clinical significance of these physiological changes, since although small changes in heart rate are unlikely to have a clinical impact, profound sinus bradycardia or bradycardia due to second- or third-degree heart block have the potential to precipitate heart failure, syncope or seizures.

\section{Evidence of cardiovascular adverse events in trials}

\section{Randomised controlled trials and meta-analyses}

We reviewed 20 randomised controlled trials (RCTs) involving donepezil, galantamine and rivastigmine (a list of the trials and summary of their findings appear in Table DS1 in the online data supplement to this article). Overall, these reported a high rate of gastrointestinal cholinergic side-effects but few cardiovascular effects.

Although all three drugs led to a significantly higher incidence of dose-related dizziness (Corey-Bloom et al, 1998; Rosler et al, 1999; Birks et al, 2000; Evans et al, 2004; Birks \& Harvey, 2006; Loy \& Schneider, 2006), its cause is multifactorial, and investigators have tended to regard dizziness as a neurological rather than a cardiovascular adverse effect.

A Cochrane review of pooled data from RCTs of all three AChE inhibitors (Birks, 2006) showed the incidence of syncope to be significantly higher in patients given an AChE inhibitor compared with those given a placebo (3.43 v. 1.87\%, $P=0.02$ ). Syncope has been noted as a serious adverse effect in active treatment with donepezil $(n=10)$ (Rogers et al, 1998; Mohs et al, 2001; Winblad et al, 2001; Seltzer et al, 2004). Half of these were considered by authors to be unrelated to donepezil and three resulted in treatment withdrawal. 


\section{Box 1 A clarification of bradycardia}

Sinus node disease

Usually caused by ischaemia, infarction or degenerative disease and results in long intervals between each atrial depolarisation. A prolonged interval between consecutive P-waves $(>2 \mathrm{~s})$ is seen on the ECG. Cardiac pacing is indicated for chronic symptomatic sinus node disease.

Atrioventricular $(A V)$ block

Affects the conduction of depolarisation between the atria and ventricles:

- First-degree AV block: every P-wave is conducted to the ventricles, but with a delay. This gives rise to a prolonged PR interval $(>0.22 \mathrm{~s})$ on ECG

- Second-degree (partial) AV block: not every P-wave is conducted to the ventricles. The three types of second-degree block are:

- Mobitz type 1 (Wenkebach): progressively lengthening PR interval until a P-wave fails to conduct to the ventricles

- Mobitz type 2: occasional P-waves are not conducted to the ventricles

- type 2:1 or 3:1 block (advanced): every second or third P-wave is not conducted to the ventricles

Mobitz type 1 second-degree AV block is the most benign. Patients with Mobitz type 2 and advanced second-degree $\mathrm{AV}$ block are at risk of progressing to complete heart block and are more likely to require cardiac pacing.

- Third-degree (complete) AV block: there is dissociation between the atria and the ventricles, and no P-waves are conducted to the ventricles. Contraction of the ventricles is maintained by a spontaneous escape rhythm, which will be broad complex (QRS $>0.12 \mathrm{~s}$ ) or narrow complex ( $\mathrm{QRS}<0.12 \mathrm{~s})$. Broad complex rhythms are usually very slow and have a high mortality, even if asymptomatic, and permanent cardiac pacing is therefore required.

Intraventricular conduction disturbance

Within the ventricles, a number of conduction disturbances can occur. Blocked conduction at the bundle of His causes AV block. Block of the right or left bundle branches causes a widening of the QRS complexes. Patients may be asymptomatic, or may complain of syncope due to intermittent complete heart block or ventricular tachycardias.

The Cochrane review also reported that pooled data from unpublished studies on the use of galantamine in people with mild cognitive impairment who were at risk of developing Alzheimer's disease found a significantly higher rate of unexplained death in those given the drug (Loy \& Schneider, 2006). As a result, it recommended that galantamine should not be used in mild cognitive impairment until there is evidence that supports or disproves the unpublished findings.

None of the RCTs we analysed showed consistent clinically significant differences between the effect of the drug and placebo on pulse, blood pressure or ECG. The higher incidence of sinus bradycardia in those treated with donepezil compared with placebo in four studies was not significant (Tariot et al, 2000; Feldman et al, 2001; Winblad et al, 2001; Black et al, 2003), and an analysis of four RCTs of rivastigmine (Morganroth et al, 2002) showed no differences in heart rate or ECG indices (including PR and QT intervals) between rivastigmine and placebo.

The incidence of ischaemic heart disease found during the trials was low and not significantly greater than in an equivalent comparison population. In addition, a number of studies demonstrated that populations with pre-existing cardiovascular disease or high rates of concurrent medication use showed no greater occurrence of cardiovascular adverse events (Kumar et al, 2000; Tariot et al, 2001; Black et $a l, 2003)$.

Birks' (2006) analysis of the limited number of head-to-head trials (Bullock et al, 2001; Wilcock et al, 2003; Jones et al, 2004; Bullock et al, 2005) revealed no consistent evidence of a difference in cardiovascular side-effect profile between the three drugs.

\section{Open-label studies}

The shortcomings of RCTs are their short duration and selective entry criteria. We must rely on reports from clinical practice and open-label studies to reveal rarer, idiosyncratic and delayed adverse effects. Numerous open-label studies have been carried out, although the weight of evidence they provide is restricted by the absence of control groups. Here we report on ten of these (for a list of the studies and the cardiovascular adverse effects reported see Table DS2 in the online data supplement to this article). 
The longest period of continuous use of $\mathrm{AChE}$ inhibitors was 4 years. Therewasnoevidence of an association between longer duration of use and increased incidence of cardiovascular adverse events such as bradycardia, syncope and dizziness. Furthermore, the cardiovascular safety profile of the AChE inhibitors was found to be the same in a 'real-world' study with few exclusion criteria (Mossello et al, 2004). We found no reports of direct drug interactions, and one trial with donepezil (Relkin et al, 2003) demonstrated that the risk of clinically important bradycardia is not significantly increased by concurrent use of betablockers, calcium antagonists or digoxin.

Astatistically insignificant mean drop in heart rate of two beats per minute and rise in the PR interval ( $3 \mathrm{~ms})$ was seen in patients taking donepezil, but neither observation was related to clinical adverse events (Froelich et al, 2004). The same study showed that patients with pre-existing first-degree heart block were no more likely to progress to seconddegree or complete heart block while on treatment, and pre-morbid cardiovascular disease was not associated with a significantly increased incidence of cardiovascular adverse effects.

\section{Case studies}

Between 1997 and 1999 in the UK, the Committee on Safety of Medicines and the Medicines Control Agency received four reports of heart block in patients receiving donepezil (one first-degree, two second-degree and one complete heart block), and 20 reports of seizure (Anonymous, 1999).

Seven cases of new-onset bradycardia presenting as syncope, and thought to be related to donepezil, have been reported (Bordier et al, 2006). Three occurred early after drug initiation; the others occurred well into treatment. On investigation, four of the patients were found to have carotid sinus hypersensitivity and three sinus node disease. Two of the patients had seizures following ventricular standstill. The causal relationship between AChE inhibitors and these events remains unclear. Cardiac pacing systems were fitted in all seven patients, and they continued donepezil.

Newby et al (2004) reported second-degree and complete heart block in a previously asymptomatic individual who suffered syncope and seizures 1 week after beginning donepezil. Similar atrioventricular rhythm disturbances associated with syncope were also reported in a French case study $3 \mathrm{~h}$ after a patient received galantamine (Brembilla-Perrot et al, 2004).

ASpanish casestudy reported a case of symptomatic sinus bradycardia on donepezil initiation, causing left ventricular failure (Calvo-Romero \& RamosSalado, 1999). The pulse rate returned to normal on discontinuation of the drug.
Finally, there has been a case report of QT interval prolongation after beginning rivastigmine, although this may have been complicated by medical comorbidity, electrolyte disturbance or polypharmacy (Walsh \& Dourish, 2002).

\section{Putting the evidence into practice}

\section{Screening}

Current guidelines from the National Institute for Health and Clinical Excellence (2001) do not include pre-treatment screening, and review authors have differed in opinion regarding the need for preinitiation and ongoing cardiovascular monitoring (Rogers et al, 2000; Aquilina, 2002; Inglis, 2002; Cummings, 2003; Rubey, 2003).

Criteria developed by Wilson \& Jungner (1968) have been used widely in assessing whether or not to implement population screening programmes, and the guidelines we outline here for managing cardiovascular risk with AChE inhibitors were developed with these criteria in mind. Wilson \& Jungner state that a screening tool should be used for conditions that cause significant morbidity and have a well-understood natural history of a preclinical asymptomatic phase that can be detected accurately and in a resource-efficient manner.

The incidence of cardiovascular adverse events with AChE inhibitors is low. Rarely, they appear to cause or unmask parasympathetically mediated bradycardias, and in a minority of people this may be associated with serious consequences. On the weight of existing evidence, intensive cardiac screening is not justified, as it produces little clinical benefit with potentially major resource implications. Furthermore, although people with sick sinus syndrome or other cardiac conduction defects may be at a theoretically greater risk of developing cardiovascular adverse effects with AChE inhibitors, additional evidence is needed to confirm this relationship in clinical practice. Currently, there is not sufficient evidence to suggest any high-risk groups that could be targeted for screening.

\section{ECGs and cardiac monitoring}

The use of ECGs before instigation of therapy is unlikely to be helpful and is not recommended. Trial evidence indicates that abnormal ECGs are not predictive of cardiovascular adverse events, and that such events also occur in those with a normal pre-treatment ECG. In addition, cardiac monitoring for $24-48 \mathrm{~h}$ could not be justified as a screening tool since it is a relatively insensitive method of detecting arrhythmias, is poorly tolerated and has high resource implications. 


\section{Drug interactions}

Although clinicians should be aware of the potential for synergy between AChE inhibitors and drugs that reduce heart rate (e.g. digoxin and beta-blockers), evidence does not support the need to routinely stop these medications before starting AChE inhibitor treatment.

\section{A clinical protocol}

The key points of our findings are summarised in Box 2. Figure 1 shows the protocol we have drawn up for managing the cardiovascular risk posed by AChE inhibitors. This is not intended to be prescriptive, but it should ensure an appropriate level of vigilance towards adverse effects to complement history-taking. Pulse rate is checked at baseline and at follow-up. We suggest monthly pulse checks during titration of the AChE inhibitor, and 6-monthly checks thereafter. Should bradycardia $(<50 \mathrm{bpm})$ be noted, even if asymptomatic, it would be sagacious to investigate its cause before beginning or continuing treatment. Those with mild bradycardia (50-60 bpm) should be monitored more frequently, to ensure that the relative bradycardia does not become symptomatic.

\section{Box 2 Key points for the clinician}

- AChE inhibitors are well tolerated and serious adverse events are rare

- AChEinhibitors are associated with rareincidences of heart block and sinus bradycardia with potentially serious consequences

- Intensive cardiovascular screening is not justified on existing evidence

- There are no high-risk groups for whom to target screening

- Vigilance is required regarding the potential for cardiovascular adverse effects with $\mathrm{AChE}$ inhibitors, and the guidelines suggested here are a means of minimising cardiovascular risk in routine clinical practice

If a patient taking an $\mathrm{AChE}$ inhibitor presents with syncope or seizures an underlying cardiovascular cause should be strongly suspected (Thompson et al, 2004). The drug should be stopped and the patient referred to a physician for further investigation. If examination reveals no causal relationship with the drug, or if a pacemaker is fitted, the drug may be restarted.

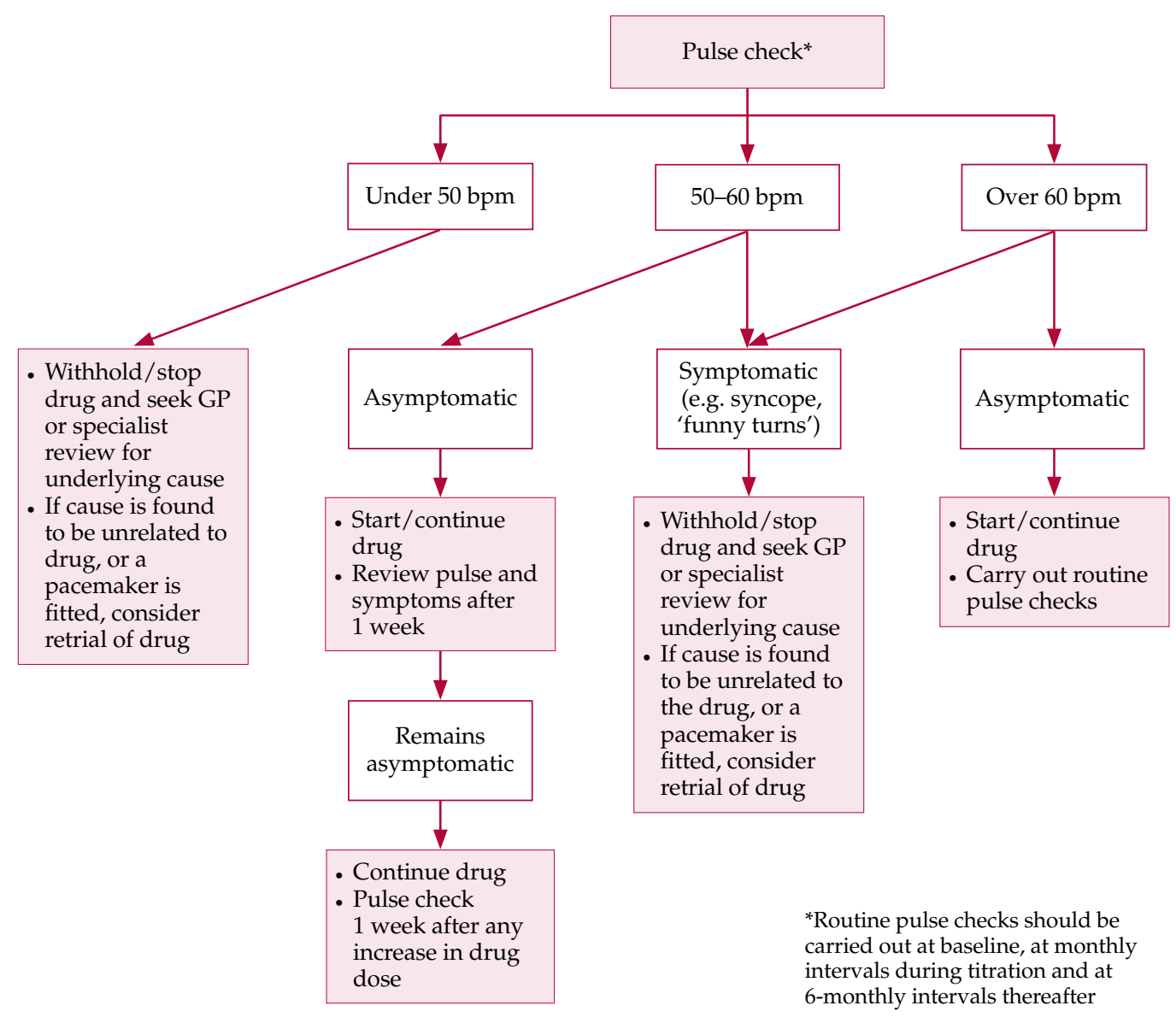

Fig. 1 Suggested guidelines for managing cardiovascular risk prior to and during treatment with acetylcholinesterase inhibitors in Alzheimer's disease. bpm, heartbeats per minute; the 'drug' means the chosen AChE inhibitor. 


\section{Box 3 Dealing with dizziness}

- Dizziness associated with AChE inhibitors is usually mild and transient

- Dizziness is usually not related to cardiovascular problems

- The cause of dizziness is often complex and multifactorial, including infections, postural hypotension and anaemia

- If dizziness is associated with a relative bradycardia (pulse rate $<60$ ) or with falls, withhold the AChE inhibitor and consider referral to an elderly care physician

- If symptoms are mild, it is probably best to continue treatment and monitor carefully. Causes unrelated to AChE inhibitors might be investigated. Simple investigations include:

- blood pressure

- postural blood pressure

- urinalysis

- routine bloods

- an ECG if available

Symptoms such as falls and dizziness often have complex and multifactorial causes, with dizziness tending to be transient and usually unrelated to cardiovascular problems. Referral to an elderly care physician for multidisciplinary assessment should be considered in these situations (Box 3).

The protocol and guidelines outlined here should be regularly audited to test their efficacy in detecting cardiovascular problems and to assess the quantity and quality of referrals generated. As new evidence comes to light, the guidelines may need to be reviewed and modified as appropriate.

\section{Declaration of interest}

None.

\section{References}

Alzheimer's Society (2004) Policy Positions: Demography. Alzheimer's Society. http://www.alzheimers.org.uk/News and_campaigns/Policy_Watch/demography.htm

Anonymous (1999) Donepezil (Aricept). Current Problems in Pharmacovigilance, 25, 7 .

Aquilina, C. (2002) A practical guide to using anti-dementia drugs. Part 2: Initiation, monitoring and termination. CME Geriatric Medicine, 4(3), 96-99.

Birks, J. (2006) Cholinesterase inhibitors for Alzheimer's disease. Cochrane Database of Systematic Reviews, issue 1. DOI 10.1002/14651858.CD005593. Wiley InterScience.

Birks, J. \& Harvey, R. J. (2006) Donepezil for dementia due to Alzheimer's disease. Cochrane Database of Systematic Reviews, issue 1. DOI: 10.1002/14651858.CD001190.pub2. Wiley InterScience.

Birks, J., Grimley Evans, J., Iakovidou, V., et al (2000) Rivastigmine for Alzheimer's disease. Cochrane Database of Systematic Reviews, issue 4. DOI: 10.1002/14651858.CD001191. Wiley InterScience.
Black, S., Roman, G. C. \& Geldmacher, D. S. (2003) Efficacy and tolerability of donepezil in vascular dementia: positive results of a 24-week, multicenter, international, randomized, placebocontrolled clinical trial. Stroke, 34, 2331-2332.

Bordier, P., Lanusee, S., Garrigue, S., et al (2006) Causes of syncope in patients with Alzheimer's disease treated with donepezil. Drugs and Aging, 22, 687-694.

Brembilla-Perrot, B., Regent, M. C., Hanesse, B., et al (2004) Troubles de la conduction auriculo-ventriculaire paroxystiques liés à la prise d'un anticholinestérasique. Archives des Maladies $d u$ Coeur et des Vaisseaux, 97, 1265-1267.

Bullock, R., Passmore, F., Potocnik, F., et al (2001) The tolerability, ease of use and efficacy of donepezil and rivastigmine in Alzheimer's disease patients: a 12-week, multinational, comparative study. Journal of the American Geriatrics Society, 49(4), S19.

Bullock, R., Touchon, J., Bergman, H., et al (2005) Rivastigmine and donepezil treatment in moderate to moderately severe Alzheimer's disease over a 2-year period. Current Medical Research and Opinion, 21, 1317-1327.

Calvo-Romero, F. M. \& Ramos-Salado, J. L. (1999) Bradicardia sinusal sintomática asociada a donepecilo. Revista de Neurologia, 28, 1070-1072.

Corey-Bloom, J., Anand, R. \& Veach, J. (1998) A randomised trial evaluating the efficacy and safety of ENA 713 (rivastigmine tartrate), a new acetylcholinesterase inhibitor, in patients with mild to moderately severe Alzheimer's disease. International Journal of Geriatric Psychopharmacology, 1, 55-65.

Cummings, J. L. (2003) Use of cholinesterase inhibitors in clinical practice - evidence-based recommendations. American Journal of Geriatric Psychiatry, 11, 131-145.

Davis, K. L., Thal, L. J., Gamzu, E. R., et al (1992) A double-blind, placebo-controlled multicenter study of tacrine for Alzheimer's disease. New England Journal of Medicine, 327, 1253-1259.

Evans, J. G., Wilcock, G. \& Birks, F. (2004) Evidence-based pharmacotherapy of Alzheimer's disease. International Journal of Neuropsychopharmacology, 7, 351-369.

Farlow, M., Gracon, S. I., Hershey, L. A., et al (1992) A controlled trial of tacrine in Alzheimer's disease. The Tacrine Study Group. JAMA, 268, 2523-2529.

Feldman, H., Gauthier, S., Hecker, J., et al (2001) A 24-week, randomized, double-blind study of donepezil in moderate to severe Alzheimer's disease. Neurology, 57, 613-620.

Fitzpatrick, A. P., Lee, R. J., Epstein, L. M., et al (1996) Effect of patient characteristics on the yield of prolonged baseline headup tilt testing and the additional yield of drug provocation. Heart, 76, 406-411.

Francis, P. T, Palmer, A. M., Snape, M., et al (1999) The cholinergic hypothesis of Alzheimer's disease: a review of progress. Journal of Neurology, Neurosurgery and Psychiatry, 66, 137-147.

Fratiglioni, L., Launer, L. J., Andersen, K., et al (2000) Incidence of dementia and major subtypes in Europe. A collaborative study of population-based cohorts. Neurological Diseases in the Elderly Research Group. Neurology, 54 (suppl. 5), S10-S15.

Froelich, L., Gertz, H. J., Heun, R., et al (2004) Donepezil for Alzheimer's disease in clinical practice - The DONALDStudy. Dementia and Geriatric Cognitive Disorders, 18, 37-43.

Giacobini, E. (1990) The cholinergic system in Alzheimer's disease. Progress in Brain Research, 84, 321-332.

Hidetaka, Y., Fukuhara,Y., Kenji, W., et al (2003) A case of acute cholinergic adverse effects induced by donepezil overdose: a follow-up of clinical course and plasma concentration of donepezil. Clinical Neurology, 43, 482-486.

Inglis, F. (2002) The tolerability and safety of cholinesterase inhibitors in the treatment of dementia. International Journal of Clinical Practice, 127 (June), 45-63.

Jackson, S., Ham, R. J. \& Wilkinson, D. (2004) The safety and tolerability of donepezil in patients with Alzheimer's disease. British Journal of Clinical Pharmacology, 58 (suppl. 1), 1-8.

Jones, R. W., Soininen, H., Hager, K., et al (2004) A multinational, randomised, 12-week study comparing the effects of donepezil and galantamine in patients with mild to moderate Alzheimer's disease. International Journal of Geriatric Psychiatry, 19, 58-67.

Knapp, J. M., Knopman, D. S., Soloman, P. R., et al (1994) A 30week randomized controlled trial of high-dose tacrine in patients with Alzheimer's disease. JAMA, 271, 985-991.

Kumar, V., Anand, R., Messina, J., et al (2000) An efficacy and safety analysis of Exelon in Alzheimer's disease patients with 
concurrent vascular risk factors. European Journal of Neurology, 7, 159-169.

Loy, C. \& Schneider, L. (2006) Galantamine for Alzheimer's disease and mild cognitive impairment. Cochrane Database of Systematic Reviews, issue 1. DOI: 10.1002/14651858.CD001747. pub3. Wiley InterScience

McLaren, A. T., Allen, J., Murray, A., et al (2003) Cardiovascular effects of donepezil in patients with dementia. Dementia and Geriatric Cognitive Disorders, 15, 183-188.

Mohs, R. C., Doody, R. S., Morris, J. C., et al (2001) A 1-year placebo-controlled preservation of function survival study of donepezil in AD patients. Neurology, 57, 481-488.

Morganroth, J., Graham, S., Hartman, R., et al (2002) Electrocardiographic effects of rivastigmine. Journal of Clinical Pharmacology, 42, 558-568.

Mossello, E., Tonon, E., Caleri, V., et al (2004) Effectiveness and safety of cholinesterase inhibitors in elderly subjects with Alzheimer's disease: a "real world" study. Archives of Gerontology and Geriatrics, 9, 297-307.

National Institute for Clinical Excellence (2001) Guidance on the Use of Donepezil, Rivastigmine and Galantamine for the Treatment of Alzheimer's Disease (NICE Technology Appraisal Guidance No. 19). NICE. http:/ / www.nice.org.uk/pdf/ALZHEIMER full_guidance.pdf

Newby, V. J., Kenny, A. \& McKeith, I. G. (2004) Donepezil and cardiac syncope: case report. International Journal of Geriatric Psychiatry, 19, 1110-1112.

Relkin, N. R., Reichman, W. E., Orazem, J., et al (2003) A large, community-based, open-label trial of donepezil in the treatment of Alzheimer's disease. Dementia and Geriatric Cognitive Disorders, 16,15-24.

Rogers, S. L., Farlow, M. R., Doody, R. S., et al (1998) A 24-week, doubleblind, placebo-controlled trial of donepezil in patients with Alzheimer's disease. Neurology, 50, 136-145.

Rogers, S. L., Doody, R. S., Pratt, R. D., et al (2000) Long-term efficacy and safety of donepezil in the treatment of Alzheimer's disease: final analysis of a US multicentre open label study. European Neuropsychopharmacology, 10, 195-203.

Rosler, M., Anand, R., Cicin-Aain, A., et al (1999) Efficacy and safety of rivastigmine in patients with Alzheimer's disease: international randomised controlled trial. BMJ, 318, 633-638.

Rubey, R. N. (2003) The cholinesterase inhibitors. Journal of Psychiatric Practice, 9, 422-430.

Savie, V., Gurun, M. S., Cavun, S., et al (1998) Cardiovascular effects of centrally injected tetrahydoraminoacridine in conscious normotensive rats. European Journal of Pharmacology, 34, 35-41.

Seltzer, B., Zolnounio, P., Nunez, M., et al (2004) Efficacy of donepezil in early-stage Alzheimer's disease - a randomized placebo-controlled trial. Archives of Neurology, 61, 1852-1856.

Shepherd, G., Klein-Schwartz, W. \& Edwards, R. (1999) Donepezil overdose: a tenfold dosing error. Annals of Pharmacotherapy, 33, 812-815

Tariot, P. N., Solomon, P. R., Morris, J. C., et al (2000) A 5-month, randomized, placebo-controlled trial of galantamine in AD. Neurology, 54, 2269-2276.

Tariot, P. N., Cummings, J. L., Katz, I. R., et al (2001) Arandomised, double-blind, placebo-controlled study of the efficacy and safety of donepezil in patients with Alzheimer's disease in the nursing home setting. Journal of the American Geriatric Society, 49, 1590-1599.

Thompson, S., Lanctot, K. L. \& Herrmann, N. (2004) Benefits and risks associated with cholinesterase inhibitor therapy in Alzheimer's disease. Expert Opinion on Drug Safety, 3, 425-440.

Walsh, E. \& Dourish, J. (2002) Prolonged QT interval with rivastigmine. British Journal of Psychiatry, 180, 466.

Wang, J., Wang, X., Irnaten, M., et al (2003) Endogenous acetylcholine and nicotine activation enhances GABAergic and glycinergic inputs to cardiac vagal neurons. Journal of Neurophysiology, 89, 2473-2481.

Wilcock, G., Howe, I., Coles, H., et al (2003) A long-term comparison of galantamine and donepezil in the treatment of Alzheimer's disease. Drugs and Aging, 20, 777-789.

Wilson, J. M. \& Jungner, G. (1968) Principles and Practice of Screening for Diseases (Public Health Paper Number 34). World Health Organization.
Winblad, B., Engedal, K., Soininen, H., et al (2001) A 1-year, randomized, placebo-controlled study of donepezil in patients with mild to moderate AD. Neurology, 57, 489-495.

\section{MCQs}

1 Cardiac adverse effects with AChE inhibitors may be caused by:

a direct cardiotoxicity

b parasympathetic stimulation via the vagal nerve

c allergy

d decreased acetylcholine levels

e cardiac ischaemia.

2 Before beginning treatment with an $\mathrm{AChE}$ inhibitor it is necessary to:

a carry out an ECG

b take a pulse check

c take blood samples to check serum electrolytes

d monitor cardiac activity continuously for $24 \mathrm{~h}$

e measure blood pressure.

3 If a patient on an AChE inhibitor presents with syncope, one should:

a refer to a GP/geriatrician for investigation of cause

b continue the drug

c measure pulse and blood pressure

$\mathrm{d}$ consider restarting the drug after a pacemaker has been fitted

e change to a different $\mathrm{AChE}$ inhibitor.

4 An AChE inhibitor should be continued if:

a the patient experiences unexplained falls

b pulse rate is over 60 and the patient has experienced recurrent syncope

c pulse rate is 45 and patient is asymptomatic

$\mathrm{d}$ the patient experiences new-onset seizures

e pulse rate is 55 and the patient is asymptomatic.

5 Cardiac conduction problems related to AChE inhibitors:

a are more common in patients with pre-existing cardiac disease

$\mathrm{b}$ are responsible for most presentations of dizziness

c occur in less than $1 \%$ of patients

$\mathrm{d}$ are usually apparent on ECGs with prolongation of QT interval

e do not present with seizures. 